\title{
Splenic infarction after reversal of warfarin using Prothrombinex-VF in a patient with a mechanical aortic valve
}

\section{"there is no \\ consensus \\ regarding \\ treatment in \\ patients with \\ mechanical \\ Clinical record \\ A 55-year-old man presented to the emergency department with a 1-day history of upper abdominal pain, haematemesis and malaena on a background of aortic valve replacement and three-vessel coronary artery bypass graft surgery. At the time of admission, his medications included warfarin (target international normalised ratio [INR], 2-3), metformin, gliclazide, ezetimibe, fenofibrate, carvedilol, ramipril, aspirin, omeprazole, duloxetine and tramadol.}

heart valves

who require

emergency

anticoagu-

lation reversal" \section{Varun Sharma
MB BS, BMedSci,} PGradSurgAnat

Talbir Singh $\mathrm{MBBS}^{2}$

Suranga Weerasooriya MBBS, MSC, FRACP2

Dharmesh Anand MB BS, MD, DNB(Cardiology), FRACP $^{2}$

IRoyal Melbourne Hospital, Melbourne, VIC.

2 Townsville Hospital, Townsville, QLD.

sharma.varun.j@ gmail.com

doi: 10.5694/mjal4.00851
Examination revealed tenderness in the right hypochondrium and epigastric regions with dark blood and malaena in the rectum. Results of biochemical investigations showed an INR of 3.2, haemoglobin level of $124 \mathrm{~g} / \mathrm{L}$ (reference interval [RI], 130-170 g/L) and urea level of $10.3 \mathrm{mmol} / \mathrm{L}$ (RI, $2.5-7.3 \mathrm{mmol} / \mathrm{L}$ ]. The patient had a history of chronic anaemia, with haemoglobin levels ranging from $120 \mathrm{~g} / \mathrm{L}$ to $125 \mathrm{~g} / \mathrm{L}$ during previous admissions in the same year. This presentation was attributed to an episode of acute upper gastrointestinal bleeding (proximal to the ligament of Treitz) exacerbated by warfarin and aspirin. Box 1 summarises the patient's investigation results during his inpatient stay.

A second episode of perirectal bleeding while the patient was in the emergency department led to the decision to transfuse one unit of packed red cells and reverse warfarin. The method for reversal was decided using clinical acumen (discussed below) and practice guidelines (Box 2), and treatment comprised administering prothrombin complex concentrate (PCC; brand name, Prothrombinex-VF) at $50 \mathrm{lU} / \mathrm{kg}$ (patient weight, $100 \mathrm{~kg}$ ) and $1 \mathrm{mg}$ of vitamin K intravenously.

After a third episode of malaena (haemoglobin, $80 \mathrm{~g} / \mathrm{L}$ ), the patient was taken urgently, in spite of poor bowel preparation, for endoscopy to identify any reversible source of bleeding. The findings of the colonoscopy were indefinite and upper gastrointestinal endoscopy did not show any active source of bleeding, so we presumed that the haemorrhage was arising from the small bowel or colon. Three hours after the endoscopy, still within the first 24 hours

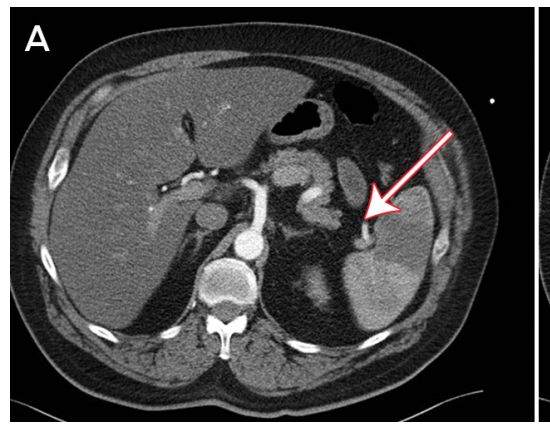

$\mathrm{T}$ his case highlights the difficulty of managing patients with a mechanical valve replacement who sustain acute major bleeding. In these patients, continuing anticoagulation exacerbates bleeding, but reversing or discontinuing it poses the risk of embolic complications from a mechanical prosthesis, some of which were seen in this of his admission, the patient developed chest pain and was found to have new ST-segment changes in leads V4-V6 and elevated serial troponins (peaking at $19 \mathrm{ug} / \mathrm{L}[\mathrm{RI},<0.04 \mathrm{ug} / \mathrm{L}$ ). Subsequent angiography revealed native triple vessel disease and graft vessel disease; however, no definite culprit lesion or thrombus were identified, so angioplasty was not considered. The likely explanation for this event was a Type 2 myocardial infarction (secondary to ischaemic imbalance due to acute bleeding) rather than an acute coronary event secondary to plaque rupture.

At this stage, we felt that the benefit of introducing antithrombotic therapy to prevent further thrombotic complications outweighed the risk of any significant gastrointestinal bleeding. Aspirin was recommenced, and heparin was commenced as bridging therapy with a target activated partial thromboplastin time (APTT) of 70 seconds.

Three days after admission, the patient developed left flank pain with a distended abdomen and guarding in the left upper quadrant. An urgent contrast computed tomography scan showed a wedge-shaped, hypodense area with absent perfusion in the lower pole of the spleen, consistent with an infarction (Figures A-C). A thrombus was identified in a small branch of the splenic artery supplying the lower pole of the spleen (Figure B). No source of the thrombus was found on transthoracic echocardiography (TTE) or transoesophageal echocardiography (TOE), and results of thrombophilia screening were negative. Although the patient received intravenous heparin anticoagulation therapy during this time, his APTT was subtherapeutic (32s). The infarction was mananged conservatively.

After the patient's symptoms resolved and there was no further bleeding, warfarin was recommenced on Day 12 of admission. The patient was discharged on Day 15 of admission after no further cardiovascular or gastrointestinal complication.
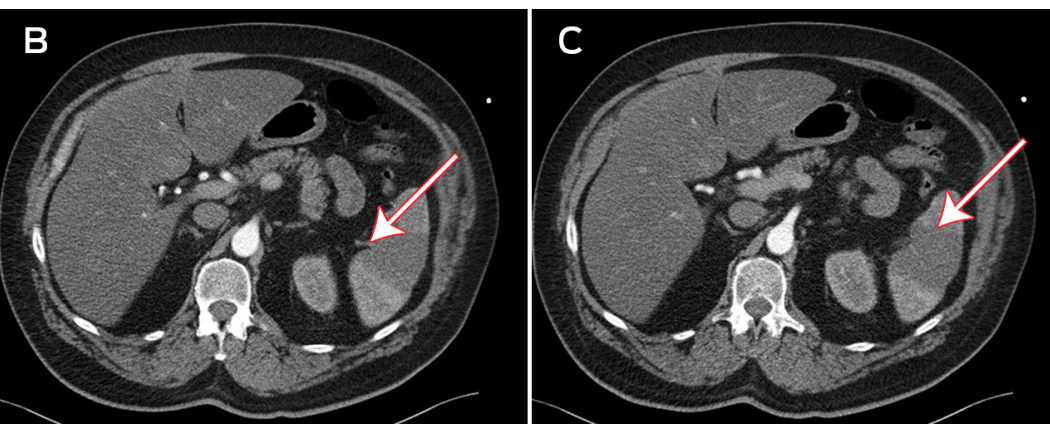

A: Contrast medium entering the inferior branch of the splenic artery, with absent perfusion in the lower pole of the spleen. B: A visible thrombus in the inferior branch of the splenic artery. C: Splenic infarction, seen as a wedge-shaped, hypodense area.

patient. Current recommendations for these patients dictate the use of prothrombin complex concentrate (PCC), but this is unproven and based only on scant evidence and a series of case reports. ${ }^{1}$

This patient had two infarctions (myocardial and splenic) that could be attributed to either an embolic source from 
Lessons from practice

- Caution needs to be exercised whenever prothrombin complex concentrate (PCC) is used in any acute setting for correction of warfarin-induced bleeding, particularly in the context of mechanical valves

- Further studies are needed to assess the safety of PCC and vitamin $\mathrm{K}$ use in the patients with mechanical heart valves.

- We suggest that complications such as the one described in this case report are rare and not substantial enough to contraindicate PCC.

the mechanical aortic valve in the setting of subtherapeutic anticoagulation or the procoagulant effects of Prothrombinex-VF.

If the mechanical valve was the source of the emboli, then, irrespective of coagulation status, a thrombus in one of the left cardiac chambers or on the mechanical valve would be expected to be seen on echocardiogram. However, both TTE and TOE did not show this. Furthermore, while anticoagulation was subtherapeutic at the time of the splenic infarction, anticoagulation with heparin had been commenced according to Queensland Health protocols. Therefore, it is unlikely that either one of the infarctions was secondary to a thrombus from the mechanical aortic valve.

The non-ST-segment myocardial infarction could potentially be attributed to several alternative factors, including a significant history of cardiovascular disease, anaemia, sympathetic stress secondary to bleeding, or coronary embolisation. However, the splenic infarction is very unlikely to have occurred spontaneously in the absence of Prothrombinex-VF.

Current guidelines for active bleeding requiring emergency reversal recommend Prothrombinex-VF, and it is preferred over fresh frozen plasma owing to the immediacy of reversal ${ }^{2,3}$ which has been well documented in selected case reports. ${ }^{4}$ Prothrombinex-VF has been shown to be a safe gold standard in warfarin-related bleeding episodes. . $, 3,5,6^{-}$

However, there is no consensus regarding treatment in patients with mechanical heart valves who require emergency anticoagulation reversal. Most case reports recommend weighing up the bleeding risk of warfarin with the thromboembolic risks of anticoagulation reversal with Prothrombinex-VF. A number of case reports have shown significant reported mortality and morbidity associated with warfarin-induced bleeding, a mortality rate of $9.5 \%-13.4 \%$, and a one in 12 chance of rebleeding,? which is why we made the decision to reverse warfarin in this patient.

Guidelines recommend vitamin K to sustain the long-term effect of warfarin reversal, but its clinical use is inconsistent. The concern with using a large dose of vitamin $\mathrm{K}(5-10 \mathrm{mg})$ is a period of warfarin reversal that outlasts the period of acute bleeding, thereby placing the patient at risk of subtherapeutic anticoagulation. For this reason, we only administered $1 \mathrm{mg}$ of vitamin K. Despite this level
1 A summary of investigations during the patient's inpatient admission

\begin{tabular}{lcccc} 
Event & $\begin{array}{c}\text { Day of } \\
\text { admission }\end{array}$ & $\begin{array}{c}\text { INR } \\
\text { (TR, 2.0-3.0; } \\
\text { normal, 1.0) }\end{array}$ & $\begin{array}{c}\text { APTT } \\
\text { (target, 70 s; } \\
\text { normal, < 40 s) }\end{array}$ & $\begin{array}{c}\mathrm{Hb} \\
\text { (RI, 130-170 } \\
\text { g/L) }\end{array}$ \\
\hline Admission & 0 & 3.2 & $\mathrm{n} / \mathrm{a}$ & 124 \\
Endoscopy & $\begin{array}{c}0 \\
\text { (about 2 h } \\
\text { after warfarin } \\
\text { reversed) }\end{array}$ & 1.5 & $\mathrm{n} / \mathrm{a}$ & 80 \\
$\begin{array}{l}\text { Myocardial } \\
\text { infarction }\end{array}$ & $\begin{array}{c}\text { (about 3 h } \\
\text { after } \\
\text { endoscopy } \\
\text { completed) }\end{array}$ & $\mathrm{n} / \mathrm{d}$ & $\mathrm{n} / \mathrm{a}$ & 88 \\
$\begin{array}{l}\text { Heparin } \\
\text { commenced }\end{array}$ & 2 & & & \\
$\begin{array}{l}\text { Splenic } \\
\text { infarction }\end{array}$ & 3 & 1.3 & 29 (initially) & 88 \\
\hline
\end{tabular}

INR = international normalised ratio. TR = therapeutic range. $\mathrm{RI}=$ reference interval. $\mathrm{APTT}=$ activated partial thromboplastin time. $\mathrm{Hb}=$ haemoglobin. $\mathrm{n} / \mathrm{a}=$ not applicable. $\mathrm{n} / \mathrm{d}=$ not done.

\section{Australasian Society of Thrombosis and Haemostasis guidelines for warfarin reversal*}

\section{Management of patients on warfarin therapy with bleeding*}

Clinical setting Recommendations and levels of evidence ${ }^{\dagger}$

\section{INR $\geqslant 1.5$ with life- Cease warfarin therapy and administer:}

threatening ${ }^{\ddagger}$

(critical organ)

bleeding

- vitamin $\mathrm{K}_{1}$ 5.0-10.0 mg IV (2C)

- and Prothrombinex-VF $50.0 \mathrm{IU} / \mathrm{kg}^{5} \mathrm{IV}$ (GPP)

- and fresh frozen plasma $150-300 \mathrm{~mL}$ (GPP)

- If Prothrombinex-VF is unavailable, administer fresh frozen plasma $15 \mathrm{~mL} / \mathrm{kg}$ (GPP)

INR $\geqslant 2.0$ with - Cease warfarin therapy and administer:

clinically

significant

bleeding (not life-

threatening)

- vitamin $\mathrm{K}_{1}$ 5.0-10.0 mg IV (2C)

- and Prothrombinex-VF 35.0-50.0 IU/kg IV (GPP) according to INR (see Box 4)

- If Prothrombinex-VF is unavailable, administer fresh frozen plasma $15 \mathrm{~mL} / \mathrm{kg}$ (GPP)

Any INR with minor bleeding

- Omit warfarin, repeat INR the following day and adjust warfarin dose to maintain INR in the target therapeutic range (2C)

- If bleeding risk is high" or INR > 4.5, consider vitamin $\mathrm{K}_{1}$, $1.0-2.0 \mathrm{mg}$ orally or $0.5-1.0 \mathrm{mg}$ IV (GPP)

INR = international normalised ratio. IV = intravenously. * Indication for warfarin therapy should be reviewed; if clinically appropriate, consider permanent cessation. † Level of evidence in parentheses in bold. Details, Box 1 . ‡ Includes intracranial bleeding. § Consider administering a Prothrombinex-VF dose less than 50.0 IU/kg when INR 1.5-1.9. 9 Recent major bleed (within previous 4 weeks) or major surgery (within previous 2 weeks), thrombocytopenia (platelet count, $<50 \times 10^{9} / \mathrm{L}$ ), known liver disease or concurrent antiplatelet therapy.

* Reproduced from the Medical Journal of Australia. ${ }^{2}$

of caution, the patient still sustained two infarctions that may have arisen from subtherapeutic anticoagulation. This suggests that further investigation is needed in administering vitamin $\mathrm{K}$ to patients with mechanical heart valves.

Competing interests: No relevant disclosures.

(c) 2015 AMPCo Pty Ltd. Produced with Elsevier B.V. All rights reserved.

References are available online at www.mja.com.au. 
1 Panduranga P, Al-Mukhaini M, Al-Muslahi M, et al. Management dilemmas in patients with mechanical heart valves and warfarin-induced major bleeding. World J Cardiol 2012; 4: 54-59.

2 Tran H, Chunilal SD, Harper PL, et al; Australasian Society of Thrombosis and Haemostasis. An update of consensus guidelines for warfarin reversal. Med J Aust 2013; 198: 198-199. https://www.mja.com.au/journal/2013/198/4/updateconsensus-guidelines-warfarin-reversal

3 Holbrook A, Schulman S, Witt DM, et al; American College of Chest Physicians. Evidence-based management of anticoagulant therapy: antithrombotic therapy and prevention of thrombosis. 9th ed. American College of Chest Physicians evidence-based clinical practice guidelines. CHEST 2012; 141 (2 Suppl): el52S-el84S.

4 Kar R, Abel E, Burcham P, Firstenberg MS. Prothrombin complex concentrate for warfarin-induced bleeding in a patient with a mechanical aortic valve. Interact Cardiovasc Thorac Surg 2013; 17: 421-422.

5 Dentali F, Ageno W, Crowther M. Treatment of coumarinassociated coagulopathy: a systematic review and proposed treatment algorithms. J Thromb Haemost 2006; 4: 1853-1863.

6 Udvardy M, Telek B, Mezey G, et al. Successful control of massive coumarol-induced acute upper gastrointestinal bleeding and correction of prothrombin time by recombinant active factor VII (Eptacog-alpha, NovoSeven) in a patient with a prosthetic aortic valve and two malignancies (chronic lymphoid leukaemia and lung cancer). Blood Coagul Fibrinolysis 2004; 15: 265-267.

7 Guerrouij M, Uppal CS, Alklabi A, Douketis JD. The clinical impact of bleeding during oral anticoagulant therapy: assessment of morbidity, mortality and post-bleed anticoagulant management. J Thromb Thrombolysis 2011; 31: 419-423. = 